\title{
Reversible posterior leukoencephalopathy syndrome induced by apatinib: a case report and literature review
}

This article was published in the following Dove Press journal: OncoTargets and Therapy

\author{
Xutong $\mathrm{Li}^{1}$ \\ Jie Chai' \\ Zhi Wang ${ }^{2}$ \\ Lin Lu' \\ Qingye Zhao' \\ Jie Zhou' \\ Fang Ju' \\ 'Department of Oncology, Second \\ Affiliated Hospital, Qingdao \\ University, Qingdao, Shandong \\ 266042, People's Republic of China; \\ 2Department of MRI, Second Affiliated \\ Hospital, Qingdao University, Qingdao, \\ Shandong 266042, People's Republic \\ of China
}

Video abstract

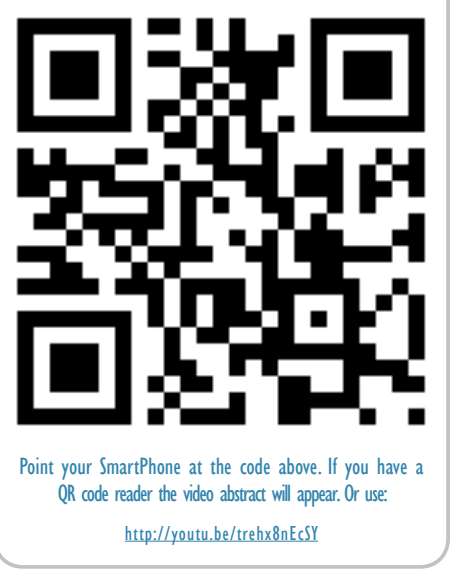

Correspondence: Fang Ju

Department of Oncology, Second Affiliated Hospital, Qingdao University,

Qingdao, Shandong 266042, People's

Republic of China

Tel +86I86 60229210

Email jufangjufang@sina.com
Abstract: Reversible posterior leukoencephalopathy syndrome (RPLS) is a rare clinicalradiologic syndrome. There are reports that RPLS may be associated with cancer therapy including some chemotherapy drugs and antiangiogenic drugs. This paper reported a case of RPLS induced by apatinib, a vascular endothelial growth factor receptor 2 (VEGFR-2) tyrosine kinase inhibitor. A 47-year-old cervical cancer patient was administered apatinib, and 3 months later, headache, dizziness, blurred vision, and hypertension appeared. She was diagnosed with RPLS by nuclear magnetic resonance imaging (MRI). After apatinib discontinuation and normotensive treatment, her symptoms completely reversed. Antiangiogenic drugs potentially damage the balance of the blood-brain barrier by directly injuring vascular endothelial cells, resulting in the occurrence of RPLS. This case is the first report of RPLS induced by apatinib.

Keywords: reversible posterior leukoencephalopathy syndrome, apatinib, antiangiogenesis, targeted therapy

\section{Case data}

A 47-year-old female patient visited the Second Affiliated Hospital of Qingdao University due to abnormal vaginal bleeding, in May 2016. She was diagnosed with poorly differentiated squamous cell carcinoma of the cervix, based on a gynecological examination and pathological biopsy. Positron emission tomography/computed tomography (PET/CT) revealed cervical cancer that extended into the vagina, uterus and bladder, with right inguinal lymph node metastasis, left external iliac vessel lymph node metastasis, lung multiple metastasis, sacral vertebral metastasis, and bilateral pleural effusion. Given that the patient had a history of left breast cancer, she received pulmonary puncture guided by CT. The pathology results of the biopsy revealed poorly differentiated carcinoma that was consistent with squamous cell carcinoma, based on immunohistochemistry. Therefore, the lung metastasis was considered to originate from cervical cancer. The patient was diagnosed with cervical squamous cell carcinoma (cT4N1M1, IV stage, according by National Comprehensive Cancer Network guidelines). A physical examination revealed an Eastern Cooperative Oncology Group (ECOG) score of 1. The left breast was absent, and the left chest wall showed postoperative changes. The vulva was normal, the vagina was clear, the cervix was seen with a cauliflower-like mass with a diameter $\sim 5 \mathrm{~cm}$, contact bleeding, the anterior fornix uteri disappeared. No positive signs were noted in other system.

Five years earlier, the patient received resection of the left breast due to breast cancer, and received six cycles of chemotherapy; she then took toremifene for 5 years. 


\section{Treatment procedure and prognosis}

The gynecologists thought the patient was beyond surgical help already. Therefore, the patient was first treated with interventional embolization due to more vaginal bleeding. Then, she received intensity-modulated radiation therapy (IMRT) $(95 \%$ plan target volume: $50.4 \mathrm{~Gy} / 1.8 \mathrm{~Gy} / 28 \mathrm{f}$ ), the target being the primary tumor, lymph-node metastatic tumor, and lymph node drainage area, and three rounds of intracavitary radiotherapy (dose total: $14 \mathrm{~Gy} / 3 \mathrm{f}$ ). When radiotherapy was over, the patient did not experience vaginal bleeding, and the local lesion was significantly reduced. The patient was unable to undergo chemotherapy due to recurrent stage II-III leucopenia during the radiotherapy. Two months later, she returned to the hospital for examination and discovered that the lung metastasis had progressed, and multiple liver metastases had appeared. Thus, the patient chose treatment with apatinib (IATAN) at $500 \mathrm{mg}$ qd. Approximately 2 weeks later, the patient experienced side effects, including diarrhea, anorexia, fatigue, hand-foot syndrome, proteinuria (+), and hypertension. The highest blood pressure was $160 / 100 \mathrm{mmHg}$. She was administered valsartan and metoprolol combined with antihypertensive treatment. Her blood pressure was maintained at $140 / 90-130 / 80 \mathrm{mmHg}$. The patient was re-examined 1 month after taking apatinib. The liver and lung metastases were reduced in size, indicating that the treatment was effective. She continued to take apatinib. After taking the medicine for 3 months, the patient experienced dizziness, headache, blurred vision, diplopia, nausea, and occasional vomiting. Her blood pressure was 150/110 $\mathrm{mmHg}$. In addition, the following test results were observed: proteinuria $(++), 2.06 \times 10^{9} / \mathrm{L}$ leukocyte count, and $53 \times 10^{9} / \mathrm{L}$ platelet count. Brain CT scan revealed a bilateral occipital lobe with patchy low-density areas considered to be bilateral occipital metastases (Figure 1A-D). Brain MRI scans revealed the following: right top temporal and occipital lobe, left parietal occipital lobe, and right cerebellar hemisphere finger-like edema that was suggestive of metastases (Figure 1E-H). Brain-enhanced MRI suggested reversible posterior leukoencephalopathy syndrome (RPLS) (Figure 1Q-T). After stopping apatinib and treating dehydration with mannitol, and hypotension via the addition of enalapril, the patient's symptoms significantly improved in 1 week. Then, she continued taking apatinib, but the dose was adjusted to half ( $250 \mathrm{mg} \mathrm{qd})$. In January 2017, the patient underwent re-examination via brain MRI, exhibiting normal characteristics (Figure 1U-X). However, 3 months later, re-examination revealed that her condition had worsened. Liver and lung metastases had obviously progressed, and multiple new metastases in the retroperitoneal lymph nodes, pleural effusion, and ascites were noted. The patient died due to liver failure 1 month later.

\section{Discussion}

Apatinib, a VEGFR-2 tyrosine kinase inhibitor, was developed by China Jiangsu Hengrui Medicine Limited, Jiangsu, People's Republic of China. The drug was approved by the China Food and Drug Administration (CFDA) in December 2014. The drug is indicated for advanced and metastatic gastric cancer/gastro-esophageal junction cancer. The main side effects include proteinuria, hypertension, hand-foot syndrome, fatigue, bleeding, cardiotoxicity and hematological toxicity (leukocytopenia, neutropenia, and thrombocytopenia). Apart from RPLS, all the other side effects that occurred in this patient were consistent with the literature and resolved after treatment.

First reported in 1996 by Judy Hinchey, ${ }^{1}$ RPLS is also referred to as posterior reversible encephalopathy syndrome (PRES). It is a clinical-radiologic syndrome. The main clinical manifestations are acute or subacute, including headache, seizures, abnormal mental behavior, visual change, and cerebral ataxia. The typical imaging changes of RPLS mainly include bilateral, symmetrical, and patchy angiogenic edema in the posterior portion of the brain (especially in the parietal occipital lobe). In addition, the frontal lobes, temporal lobes, basal ganglia, brainstem, and cerebellum can also be involved. The lesion exhibited a low-density area on CT, a low signal on T1Wl MRI, and a high $\mathrm{T} 2 \mathrm{Wl}$ and fluid-attenuated inversion recovery signal. About $15 \%-30 \%$ of patients in the diffusion-weighted imaging (DWI) sequence can be observed in the diffusion limitation of a small area within the larger angiogenic edema region, indicating irreversible structural damage and clinically incomplete recovery. ${ }^{2}$ The prognosis for the patient with this disease, is good. Most patients' neurological symptoms can be completely reversed. However, if a correct diagnosis and timely treatment are not achieved, vasogenic edema may progress to cytotoxic edema. Patients may experience irreversible neurological damage and even death. The mortality rate due to RPLS is $\sim 15 \%{ }^{3}$

Variable causes of RPLS have been noted. The condition is more commonly noted in the context of the following conditions: malignant hypertension, eclampsia, serious kidney diseases, immunosuppressive therapy after organ transplantation, systemic lupus erythematosus, and malignant tumor treatment. Reports regarding RPLS are not uncommon 

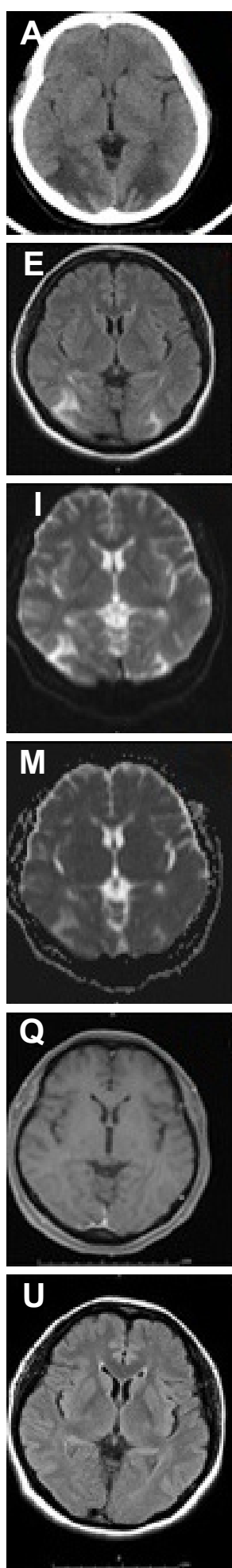
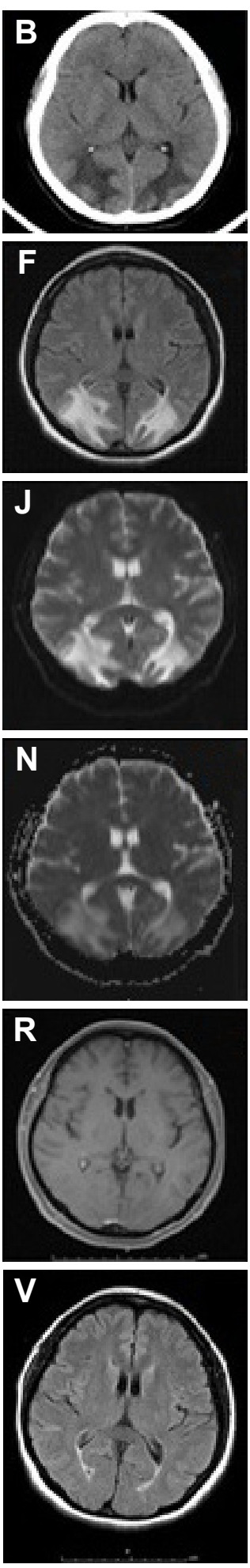
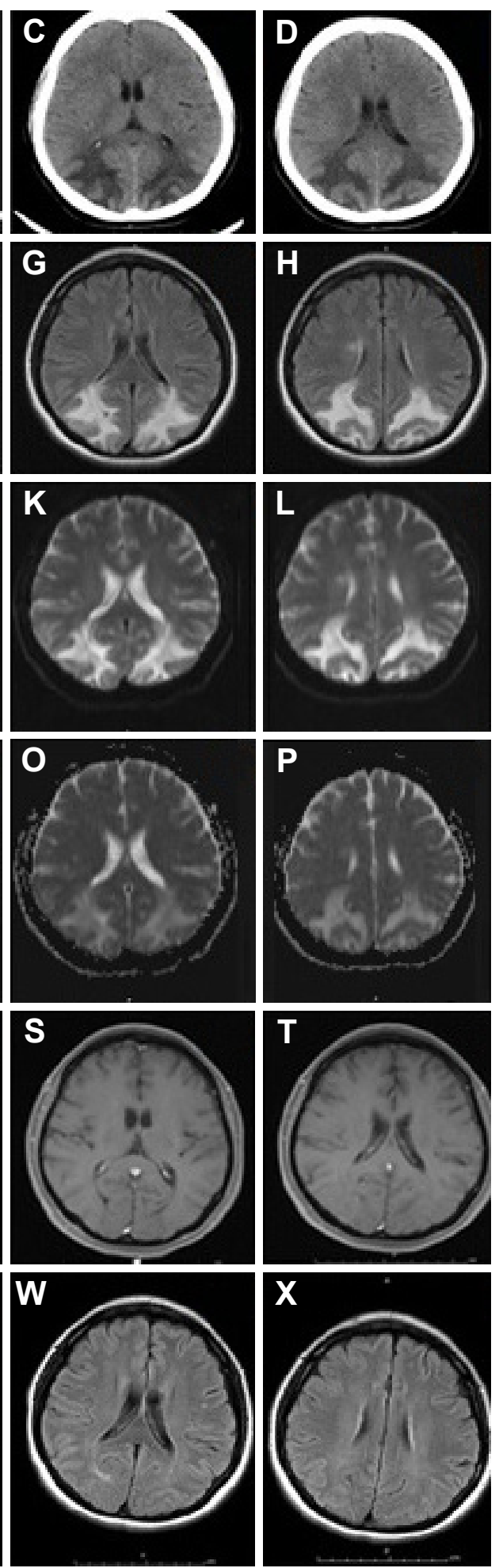

Figure I Imaging manifestations of the patient.

Notes: (A-D) Initial CT, the bilateral apical occipital lobe exhibited a flaky, low-density area, and the diagnosis of bilateral occipital lobe metastases was considered. (E-H) Initial FLAIR MRI, the right parietal temporal occipital lobe, left occipital lobe, and right cerebellar hemisphere exhibited index finger-like structures, long TI, long T2, high FLAIR signal, and fuzzy boundaries. A diagnosis of metastases was considered. Enhanced MRI examination was recommended. (I-L) Initial DWI MRI, there were no obvious abnormalities on DWI. (M-P) Initial ADC MRI, flaky high signal in occipital lobes and parietal lobes. (Q-T) Initial TI enhancement MRI, the right cerebellar hemisphere, right temporal occipital lobe, and left apical occipital vein shape exhibited no obvious enhancement and still revealed a low signal. A diagnosis of RPLS was considered, and re-examination after treatment was suggested. (U-X) FLAIR MRI I month after treatment, the white matter patch in the posterior brain improved. Abbreviations: ADC, apparent diffusion coefficient; CT, computed tomography; DWI, diffusion-weighted imaging; FLAIR, fluid-attenuated inversion recovery; MRI, magnetic resonance imaging. 
during cancer therapy ${ }^{4}$ including some chemotherapy drugs, such as paclitaxel, ${ }^{5}$ platinum, ${ }^{6,7}$ fluorouracil and irinotecan, ${ }^{6,7}$ pemetrexed and gemcitabine. ${ }^{8,9}$ In particular, reports have been published regarding RPLS induced by antiangiogenic drugs, such as bevacizumab, ${ }^{10-12}$ sorafenib, ${ }^{13}$ sunitinib,${ }^{14}$ pazopanib, ${ }^{15}$ axitinib, ${ }^{16}$ and cediranib. ${ }^{17}$ This case is the first report of RPLS induced by apatinib.

Currently, the pathogenesis of RPLS remains controversial. The following mechanism is thought to be involved. Blood pressure suddenly increases, and various pathogenic factors lead to intracranial vascular endothelial dysfunction or injury and fluid retention, subsequently causing the failure and breakthrough of the autoregulatory cerebral vascular mechanism. Local cerebral vasodilatation formation causes vasodilatation and vasoconstriction, resulting in excessive brain perfusion, blood-brain barrier damage, and entering of plasma into the surrounding glial cells and interstitial areas via capillary wall leakage. These symptoms produce subsequent vasogenic brain edema or petechial hemorrhage, leading to the symptoms of RPLS. ${ }^{18-23}$ Cytotoxic chemotherapy drugs and antiangiogenic drugs may directly damage the cerebral vascular endothelium, and hypertension is one of the common side effects of VEGFR-TKI. These factors destroy the blood-brain barrier balance and cause RPLS. In clinical practice, oncologists typically know little about this disease, so a diagnosis from a radiologist is critical. The common differential diagnoses include demyelinating disease, venous sinus thrombosis, and cerebral infarction. This case is mainly identified with brain metastases. There was no definite tumor in the brain, and the symptoms and images were reversible after treatment, which were crucial points of the differential diagnosis. Although RPLS is not mentioned in the medicine instruction for apatinib, the author thought the RPLS was induced by apatinib through medication history, the images of brain and the course of the disease, and the pathogenic mechanism which is similar to other antiangiogenic drugs.

Although RPLS induced by a targeted drug, such as bevacizumab, is rare, given the gradual popularization of targeted drugs and gradual recognition of RPLS, the incidence of RPLS may increase in the future. RPLS can be classified as an acute complication associated with cancer treatment, and the diagnosis and treatment of RPLS can be standardized. A diagnosis can be obtained through clinical presentation and typical MRI images. The principle of treatment involves stopping the drug immediately and providing aggressive treatment for normotension. The prognosis of the vast majority of cases is good. There are few reports on whether RPLS will relapse if the patient continues to take the medicine. In this case, the patient continued to take apatinib at half the dose 1 week later. She did not experience RPLS again. The cause was unknown. Perhaps it was an open question.

\section{Acknowledgment}

The patient's husband provided written informed consent for publication of the case details and any accompanying images.

\section{Disclosure}

The authors report no conflicts of interest in this work.

\section{References}

1. Hinchey J, Chaves C, Appignani B, et al. A reversible posterior leukoencephalopathy syndrome. $N$ End J Med. 1996;334(8):494-500.

2. Fugate JE, Rabinstein AA. Posterior reversible encephalopathy syndrome: clinical and radiological manifestations, pathophysiology, and outstanding questions. Lancet Neurol. 2015;14(9):914-925.

3. Lee VH, Wijdicks EF, Manno EM, Rabinstein AA. Clinical spectrum of reversible posterior leukoencephalopathy syndrome. Arch Neurol. 2008;65(2):205-210.

4. Tlemsani C, Mir O, Psimaras D, et al. Acute neurovascular events in cancer patients receiving anti-vascular endothelial growth factor agent: Clinical experience in Paris University Hospitals. Eur J Cancer. 2016;66:75-82.

5. Singer S, Grommes C, Reiner AS, Rosenblum MK, Deangelis LM. Posterior reversible encephalopathy syndrome in patients with Cancer. Oncologist. 2015;20(7):806-811.

6. Femia G, Hardy TA, Spies JM, et al. Posterior reversible encephalopathy syndrome following chemotherapy with oxaliplatin and a fluoropyrimidine: a case report and literature review. Asia Pac J Clin Oncol. 2012;8(2):115-122.

7. Dedić Plavetić N, Rakušić Z, et al. Fatal outcome of posterior "reversible" encephalopathy syndrome in metastatic colorectal carcinoma after irinotecan and fluoropyrimidine chemotherapy regimen. World J Surg Oncol. 2014;20(12):264.

8. Xie C, Jones VT. Reversible posterior leukoencephalopathy syndrome following combinatorial cisplatin and pemetrexed therapy for lung cancer in a normotensive patient: a case report and literature review. Oncol Lett. 2016;11(2):1512-1516.

9. Maeda T, Kikuchi E, Matsumoto K, et al. Gemcitabine and cisplatin chemotherapy induced reversible posterior leukoencephalopathy syndrome in a bladder cancer patient. Int J Clin Oncol. 2010;15(5): 508-811.

10. Ozcan C, Wong SJ, Hari P. Reversible posterior leukoencephalopathy syndrome and bevacizumab. N Engl J Med. 2006;354(9):980-982.

11. Massey J. Posterior reversible encephalopathy syndrome (PRES) with sub-arachnoid haemorrhage after bevacizumab and 5-FU. J Clin Neurosci. 2017;40:57-59.

12. Sclafani F, Gullo G, Mezynksi J, Collins C, Crown J. Reversible posterior leukoencephalopathy syndrome and bevacizumab in breast cancer. $J$ Clin Oncol. 2012;30(26):257-259.

13. Furubayashi N, Negishi T, Iwai H, Nagase K, Nakamura M. Sorafenibinduced reversible posterior leukoencephalopathy in patients with renal cell carcinoma: a report of two cases. Mol Clin Oncol. 2017;7(2): 281-284.

14. Saraceno L, Ricigliano VAG, Cavalli M, et al. Posterior reversible encephalopathy syndrome after long-term treatment with low-dose sunitinib: a case report. Neurol Sci. 2017;38(6):1119-1121. 
15. Chelis L, Souftas V, Amarantidis K, et al. Reversible posterior leukoencephalopathy syndrome induced by pazopanib. BMC Cancer. 2012; 22(12):489.

16. Levy A, Benmoussa L, Ammari S, Albiges L, Escudier B. Reversible posterior leukoencephalopathy syndrome induced by axitinib. Clin Genitourin Cancer. 2014;12(1):33-34.

17. Kim CA, Price-Hiller J, Chu QS, et al. Atypical reversible posterior leukoencephalopathy syndrome (RPLS) induced by cediranib in a patient with metastatic rectal cancer. Invest New Drugs. 2014;32(5): 1036-1045.

18. Garg RK. Posterior leukoencephalopathy syndrome. Postgrad Med J. 2001;77:24-28.

19. Karia SJ, Rykken JB, McKinney ZJ, Zhang L, McKinney AM. Utility and significance of gadolinium-based contrast enhancement in posterior reversible encephalopathy syndrome. AJNR Am J Neuroradiol. 2016;37(3):415-422.
20. Rykken JB, McKinney AM. Posterior reversible encephalopathy syndrome. Semin Ultrasound CT MR. 2014;35(2):118-135.

21. Dinsdale HB, Robertson DM, Haas RA. Cerebral blood flow in acute hypertension. Arch Neurol. 1974;31(2):80-87.

22. Truwit CL, Denaro CP, Lake JR, DeMarco T. MR imaging of reversible cyclosporin A-induced neurotoxicity. AJNR Am J Neuroradiol. 1991; 12(4):651-659.

23. Shankar J, Banfield J. Posterior reversible encephalopathy syndrome: a review. Can Assoc Radiol J. 2017;68(2):147-153.

\section{Publish your work in this journal}

OncoTargets and Therapy is an international, peer-reviewed, open access journal focusing on the pathological basis of all cancers, potential targets for therapy and treatment protocols employed to improve the management of cancer patients. The journal also focuses on the impact of management programs and new therapeutic agents and protocols on

\section{Dovepress}

patient perspectives such as quality of life, adherence and satisfaction. The manuscript management system is completely online and includes a very quick and fair peer-review system, which is all easy to use. Visit http://www.dovepress.com/testimonials.php to read real quotes from published authors.

Submit your manuscript here: http://www.dovepress.com/oncotargets-and-therapy-journal 